\title{
Sophoricoside from Sophora japonica ameliorates allergic asthma by preventing mast cell activation and CD4+ T cell differentiation in ovalbumin-induced mice
}

\author{
Byung-Hak Kim ${ }^{1}$, Dong-Gu Lee ${ }^{2}$, and Sanghyun Lee ${ }^{2}$ \\ ${ }^{1}$ UNDBIO Co. Ltd. \\ ${ }^{2}$ Chung-Ang University
}

April 28, 2020

\begin{abstract}
Background and Purpose: Asthma is a chronic inflammatory lung disorder with continuously increasing prevalence worldwide. Novel strategies are needed to prevent or improve asthma. The aim of this study was to investigate the effects of sophoricoside from Sophora japonica on allergic asthma. Experimental Approach: Sophoricoside was isolated from the mature seeds of S. japonica. Allergic asthma and passive cutaneous anaphylaxis (PCA) models were generated by ovalbumin (OVA) treatment or antibody-allergen reaction in mice. Allergic and asthmatic symptoms, immune cell infiltration, and the amounts of histamine, lipid metabolites, and pro-inflammatory cytokines were determined, and in vitro CD4+ T cell differentiation was performed. Key Results: The mature seeds of S. japonica contain a large amount of sophoricoside. Sophoricoside reduced allergic and asthmatic symptoms by suppressing airway inflammation and antibody-antigen reaction in mouse models. In particular, sophoricoside suppressed immune cell recruitment into the airway lumens of the lungs and production of pro-inflammatory cytokines in the bronchoalveolar lavage fluid (BALF) of OVA-induced mice. It also decreased the amounts of histamine and lipid metabolites released in OVA-induced mice and antibody-antigen stimulated mast cells. In addition, sophoricoside decreased differentiation of naïve CD4+ T cells into T helper type 1 (Th1), Th2, and Th17 cells. Conclusions and Implications: Sophoricoside from S. japonica inhibited allergic asthma by suppressing mast cell activation and CD4+ T cell differentiation. Thus, it is a promising candidate molecule for the treatment of allergic asthma. Keywords Airway inflammation; Allergic asthma; CD4+ T cell; Mast cell; Ovalbumin; Sophora japonica; Sophoricoside
\end{abstract}

\section{Keywords}

Airway inflammation; Allergic asthma; CD4 ${ }^{+}$T cell; Mast cell; Ovalbumin; Sophora japonica ; Sophoricoside

\section{INTRODUCTION}

Allergic diseases are associated with hypersensitivity to external substances and are among the most common chronic diseases worldwide. Common allergic diseases include bronchial asthma, allergic rhinitis, allergic conjunctivitis, atopic dermatitis, and food allergies. Allergic asthma is a complex chronic airway inflammatory disease that causes sporadic breathing difficulties. It causes aberrant immune responses with inhalation of allergens such as house dust mites, cockroaches, animal allergens, pollens, and indoor mold. Allergic asthma has variable and recurring symptoms, such as reversible airflow obstruction, airway hyperresponsiveness (AHR), easily triggered bronchospasms, excessive T cell-mediated immune responses, mucus hypersecretion, and airway remodeling in the lung (Fahy, 2015; Ray et al., 2016).

Recent studies have reported that allergic asthma is an aberrant anaphylactic immune response that involves a variety of immune cells, including eosinophils, T cells, B cells, mast cells, and airway epithelial cells 
(Marshall et al., 2018). In particular, activated type $2 \mathrm{~T}$ helper (Th2) CD4 ${ }^{+}$cells release type 2 cytokines, such as interleukin (IL)-4, IL-5, and IL-13, to stimulate the production of immunoglobulin E (IgE). IgE plays an important role in inducing asthmatic inflammation by promoting mast cell activation, goblet cell hyperplasia, excessive mucus secretion, eosinophil infiltration, and AHR (Fahy, 2015; Ray et al., 2016). Although the incidence of asthma has steadily increased over the past few decades, current therapeutic approaches still largely rely on anti-inflammatory corticosteroids and bronchodilators (Papi et al., 2018). Despite advances in diagnosis and treatment, asthma remains a serious global health problem, and many patients do not have properly controlled disease (Quirt et al., 2018; Zinellu et al., 2019). In fact, numerous immune cells and immune cell-driven mediators contribute to the exacerbation and progression of allergic asthma. Targeting these immune cells and mediators is a potential modality for the treatment of asthma.

Sophoricoside is an isoflavone glycoside isolated from Sophora japonica, a plant of the Leguminosae family. Sophoricoside was initially isolated in 1996, when its hormonal effect was reported (Gabor, 1961). However, few biological and pharmacological activities of sophoricoside have been reported. Its activities include IL-5 inhibitor (Min et al., 1999; Jung et al., 2003), anti-inflammation and immunosuppression (Kim et al., 2003; Kim et al., 2013; Lee et al., 2013), estrogenic effect (El-Halawany et al., 2010), modulation of lipogenesis and glucose consumption (Wu et al., 2013), hepatoprotection ( $\mathrm{Li} \& \mathrm{Lu}, 2018$ ), and anti-osteoporosis (Abdallah et al., 2014). Interestingly, sophoricoside inhibited the bioactivities of IL-3, IL-5, and IL-6 in BAF/BO3, Y16, and MH60/BSF-2 cells, but not those of IL-1 $\beta$, tumor necrosis factor-alpha (TNF- $\alpha$ ), and granulocytemacrophage colony-stimulating factor (GM-CSF) in A375.S2, WEHI-164, and TF-1 cells (Yun et al., 2000). Sophoricoside also inhibited IL-6 and cyclooxygenase (COX)-2 activities, but not the activities and expression of IL-1 $\beta, \mathrm{TNF}-\alpha$, and inducible nitric oxide synthase (iNOS) or the production of reactive oxygen species (ROS) and nitric oxide (NO) in RAW264.7 cells (Kim et al., 2003). Specifically, IL-3, IL-5, and IL-6 are Th2 cell-driven pro-inflammatory cytokines and are related to allergy and asthma induced by antigen-mediated immune response (Lambrecht, Hammad, \& Fahy, 2019).

Based on this evidence, sophoricoside was isolated from the mature seeds of S. japonica, and its anti-allergic and anti-asthmatic effects were demonstrated using in vivo mouse models. Sophoricoside ameliorated allergic asthma by preventing mast cell activation and differentiation of $\mathrm{CD} 4^{+} \mathrm{T}$ cells in ovalbumin (OVA)-induced mice.

\section{METHODS}

\subsection{Plant materials and sample preparation}

Dried mature seeds, leaves, stems, and roots of S. japonica were obtained from National Baekdudaegan Arboretum, 2019. The voucher specimen was identified by Dr. Y. S. Kim, National Baekdudaegan Arboretum and deposited at the herbarium of the Natural Product Institute of Science and Technology, Chung-Ang University, Anseong, Republic of Korea. Five grams of dried mature seeds, leaves, stems, and roots of $S$. japonica were repeatedly extracted with $70 \%$ ethanol $(100 \mathrm{~mL} \times 3)$ at $80^{\circ} \mathrm{C}$ for $3 \mathrm{~h}$ and then filtered using $0.45 \mu \mathrm{m}$ of polyvinylidene fluoride (PVDF) membrane filter for high-performance liquid chromatography (HPLC).

\subsection{Isolation and HPLC conditions for sophoricoside analysis from S. japonica}

Sophoricoside was isolated from the mature seeds of $S$. japonicaby repeated column chromatography. To quantify sophoricoside, an Agilent 1260 Infinity II Quat Pump (CA, USA) was used. Quantitative analysis was performed using a reverse-phase system. Separation was conducted on an INNO C18 column $(25 \mathrm{~cm}$ $\times 4.6 \mathrm{~mm}, 5 \mu \mathrm{m})$. Column temperature was maintained at $30^{\circ} \mathrm{C}$, and the flow rate was set at $1 \mathrm{~mL} / \mathrm{min}$. The mobile phase was comprised of (A) water (0.5\% acetic acid) and (B) acetonitrile, and the elution was conducted in a gradient system: $92 \%$ (A)-8\% (B) for $2 \mathrm{~min}$; reduced to $90 \%$ (A) for $25 \mathrm{~min}$; reduced to $70 \%$ (A) for $23 \mathrm{~min}$; reduced from $70 \%$ to $10 \%$ for $2 \mathrm{~min}$; reduced from $10 \%$ to $0 \%$ for $8 \mathrm{~min}$; and increased from $0 \%$ to $92 \%$ (A) for $5 \mathrm{~min}$. The injection volume was $10 \mu \mathrm{L}$, and the UV absorbance was recorded at $270 \mathrm{~nm}$. 


\subsection{Reagents and cell culture}

HPLC grade solvents (acetonitrile and water) were purchased from Samchun Pure Chemicals (Pyeongtaek, Korea). Chicken egg ovalbumin (OVA), dinitrophenol (DNP)-specific monoclonal IgE, DNP-human serum albumin (HSA), and Evans blue were purchased from Sigma-Aldrich (St. Louis, MO, USA). Aluminum hydroxide (Imject ${ }^{\mathrm{TM}}$ ) were obtained from Thermo Fisher Scientific (Rockford, IL, USA). All other chemicals were obtained from Sigma-Aldrich unless otherwise noted. The human mast cell line HMC-1 was grown in Iscove's Modified Dulbecco's Medium (IMDM) supplemented with 10\% fetal bovine serum (FBS) and 1\% penicillin/streptomycin (Invitrogen, Carlsbad, CA, USA) at $37^{\circ} \mathrm{C}$ under humidified atmosphere containing $5 \%$ carbon dioxide $\left(\mathrm{CO}_{2}\right)$.

\subsection{OVA-induced allergic airway inflammation}

BALB/c mice (6-8 week old) were used for experimental study after one week of acclimation. OVA-induced allergic airway inflammation was generated using previously described modifications (Casaro et al., 2019). Briefly, mice were sensitized by intraperitoneal injection on days 0 and 14 with $200 \mu \mathrm{L}$ saline suspension containing $50 \mu \mathrm{g}$ OVA emulsified in $1.6 \mathrm{mg}$ aluminum hydroxide. After one week, mice were further challenged by intranasal inhalation of 1\% OVA aerosol from days 21 to 24 for 30 min. Dimethyl sulfoxide (DMSO, 1\%) in phosphate-buffered saline (PBS) or sophoricoside $(3$ or $30 \mathrm{mg} / \mathrm{kg}$ ) was intraperitoneally administered an hour before OVA challenge from days 20 to 25 . Mice were sacrificed $24 \mathrm{~h}$ after final treatment, and blood and organs were collected for further experiments. All animal care and studies were conducted under specific pathogen-free conditions in accordance with the guidelines of the Institutional Animal Care and Use Committee at Chung-Ang University (Approval number: 2019-00113).

\subsection{Nasal rubbing}

Nasal rubbing was assessed $24 \mathrm{~h}$ after final administration of sophoricoside. A single event was considered each time a mouse rubbed or touched the area near its nose with its forefoot. Each mouse was acclimatized with an observation cage (one mouse/cage) for $10 \mathrm{~min}$ before the experiment, and nasal rubbing was counted for $10 \mathrm{~min}$.

\subsection{Airway hyperresponsiveness (AHR)}

Airway hypersensitivity was measured by methacholine-induced airway contraction using a plethysmograph (OCP-3000, Allmedicus, Anyang, Korea) after $24 \mathrm{~h}$ of final OVA inhalation. Each mouse was put into the body record box of whole-body plethysmograph nebulized with $10-80 \mathrm{mg} / \mathrm{mL}$ methacholine for 3 min at each increasing concentration. The airway term was measured for $3 \mathrm{~min}$ after inhalation with a haze for $3 \mathrm{~min}$ while increasing the methacholine concentration. Enhanced pause (Penh) is an indicator of the airway term obtained by the following formular:

$\mathrm{Penh}=(\mathrm{Te} / \mathrm{Tr}-1) \times \mathrm{PEF} / \mathrm{PIF}$

Where Te is expiratory time (sec), Tr is relaxation time (sec), PEF is peak expiratory flow (mL/sec), and PIF is peak inspiratory flow $(\mathrm{mL} / \mathrm{sec})$.

\subsection{Bronchoalveolar lavage fluid (BALF) and inflammatory cells}

BALF was collected by washing the airway lumens of the lungs three times with $1 \mathrm{~mL}$ ice cold PBS instilled into a tracheal polyethylene catheter. The fluid was centrifuged at $2,000 \mathrm{rpm}$ for $5 \mathrm{~min}$ at $4^{\circ} \mathrm{C}$. The supernatant was collected to determine histamine contents released from airway mast cells. Collected cell pellets were used to count the number of total cells and inflammatory cells, including macrophages, eosinophils, neutrophils, and lymphocytes. Total cells were counted using a hemocytometer, and differential inflammatory cells were counted using a cytospin at $500 \mathrm{rpm}$ for $5 \mathrm{~min}$ followed by Diff-Quick (Sysmex Corporation, Kobe, Japan) or Wright-Giemsa staining (Sigma-Aldrich) according to the manufacturer's protocol. Types of inflammatory cells were determined with a differential cell counter (Hwashin Technology, Seoul, Korea) using standard morphologic criteria (Saltini et al., 1984). 


\subsection{Enzyme-linked immunosorbent assay (ELISA)}

Samples were prepared from mice serum, BALF supernatant, and cell culture media. Total IgE, OVAspecific immunoglobulins (IgE, IgG1, and IgG2a), cytokines (IFN- $\gamma$, TNF- $\alpha$, IL-4, IL-5, IL-13 and IL-17), histamine, prostaglandin $\mathrm{D}_{2}\left(\mathrm{PGD}_{2}\right)$ and leukotrienes $\left(\mathrm{LTs}, \mathrm{LTB}_{4}\right.$ and $\left.\mathrm{LTC}_{4}\right)$ were determined using an ELISA kit according to the manufacturer's protocol. Kits were obtained from Invitrogen, Cayman (Ann Arbor, MI, USA), BioLegend (San Diego, CA, USA), R\&D Systems (Minneapolis, MN, USA), and Abcam (Cambridge, UK), respectively. Absorbance was measured using a Victor Nivo Multimode Microplate Reader (PerkinElmer, Boston, MA, USA).

\subsection{Fluorescence-activated cell sorting (FACS)}

Draining lymph node cells were prepared from the lymph nodes of OVA-induced BALB/c mice ground into single cells. To determine the population of IL-4- and IFN- $\gamma$-positive cells, draining lymph node cells or BALF cells were stained with APC-conjugated anti-IL-4 antibody, FITC-conjugated anti-IFN- $\gamma$ antibody, or PE-conjugated anti-CD4 antibody (BD Pharmingen, San Jose, CA, USA) using a Cytofix/Cytoperm kit (BD Biosciences, San Jose, CA, USA) according to the manufacturer's protocol. In addition, IgE- and IgG1positive cells were stained with FITC-conjugated anti-IgE antibody or PE-conjugated anti-IgG1 antibody (BD Pharmingen). Stained cells were detected by flow cytometry using the BD LSRFortessa ${ }^{\mathrm{TM}}$ cell analyzer (BD Biosciences).

\subsection{Quantitative real-time polymerase chain reaction (qRT-PCR)}

Total RNA was isolated using TRIzol Reagent (Invitrogen), and first-strand cDNA was synthesized from 1 $\mu \mathrm{g}$ total RNA using a QuantiTect Rereverse Transcription Kit (Qiagen, Valencia, CA, USA). qRT-PCR was performed using a QuantiFast SYBR Green PCR master mix (Qiagen) with an CFX96 Real-Time System (Bio-Rad, Hercules, CA, USA). The data were analyzed by comparative $\mathrm{C}_{t}$ quantification, and the value for each sample was normalized to the value for the housekeeping $G A P D H$ gene. Primer sets for T-bet, GATA-3, ROR $\gamma$ t, IFN- $\gamma$, IL-4, IL-17A, and GAPDH were obtained from Qiagen. The amplification program consisted of 1 cycle of $95^{\circ} \mathrm{C}$ for 5 min followed by 25 cycles of $96^{\circ} \mathrm{C}$ for $10 \mathrm{sec}, 56^{\circ} \mathrm{C}$ for $20 \mathrm{sec}$, and $72^{\circ} \mathrm{C}$ for $20 \mathrm{sec}$.

\subsection{In vitro differentiation of $\mathrm{CD}^{+} \mathrm{T}$ cells}

Naïve $\mathrm{CD} 4^{+} \mathrm{T}$ cells were purified from spleens and lymph nodes of C57BL/6 mice by negative selection using T-cell enrichment columns (R\&D Systems) and mouse $\mathrm{CD} 4^{+} \mathrm{T}$ cell isolation kits (Miltenyi Biotech, Auburn, CA, USA). In vitrodifferentiation of $\mathrm{CD} 4^{+} \mathrm{T}$ cells was performed as previously described (Kim et al., 2014; Lee et al., 2016). Briefly, cells were activated by plate-bound anti-CD3 antibody in a 96-well plate (BD Biosciences) and soluble anti-CD28 $(2 \mu \mathrm{g} / \mathrm{mL})$ antibody. The cells were differentiated under Th1 polarizing conditions $(10 \mathrm{ng} / \mathrm{mL}$ IL-12 and $10 \mu \mathrm{g} / \mathrm{mL}$ anti-IL-4 antibody), Th2 polarizing conditions (20 $\mathrm{ng} / \mathrm{mL}$ IL-4 and $10 \mu \mathrm{g} / \mathrm{mL}$ anti-IFN- $\gamma$ antibody), or Th17 polarizing conditions $(20 \mathrm{ng} / \mathrm{mL}$ IL-6, $10 \mathrm{ng} / \mathrm{mL}$ TGF- $\beta, 10 \mu \mathrm{g} / \mathrm{mL}$ anti-IFN- $\gamma$ antibody and $10 \mu \mathrm{g} / \mathrm{mL}$ anti-IL-4 antibody). All cytokines and antibodies used for $\mathrm{CD} 4^{+} \mathrm{T}$ cell differentiation were obtained from BD Biosciences.

\subsection{Passive cutaneous anaphylaxis (PCA)}

C57BL/6 mice (6-8 week old) were locally sensitized with an intradermal injection of anti-DNP-specific IgE (100 ng/ear) into the right ear followed $24 \mathrm{~h}$ later by topical treatment with sophoricoside (3 or 30 $\mathrm{mg} / \mathrm{kg}$ ). Control mice were injected with the same volume of PBS and topically treated with $0.1 \%$ DMSO. One hour later, the mice were intravenously challenged with soluble antigen (100 $\mu$ g DNP-HSA) in 100 $\mu \mathrm{L}$ PBS containing 5\% Evans blue dye. One hour after antigen challenge, the dye was extracted from each dissected ear in $0.8 \mathrm{~mL}$ formamide at $65^{\circ} \mathrm{C}$ for overnight. The amount of extracted dye was measured spectrophotometrically at $620 \mathrm{~nm}$ and calculated from a standard curve established with known amounts of dye.

\subsection{Histology}


Tissues were fixed in $10 \%$ neural buffered formalin for $24 \mathrm{~h}$. After dehydration, the tissues were embedded in paraffin, cut into $5 \mu \mathrm{m}$ thick sections, and stained with hematoxylin and eosin (H\&E). To determine histopathologic features, digital images were obtained using LAS microscope software (Leica Microsystems, Wetzlar, Germany).

\subsection{Cell viability}

Cells were seeded in 96-well plates and incubated in culture medium overnight. Cells were then further incubated with either vehicle alone or various concentrations of sophoricoside for 24-72 h. Cell viability was measured at $450 \mathrm{~nm}$ using a Victor Nivo Multimode Microplate Reader (PerkinElmer) after further incubation for $2-4 \mathrm{~h}$ at $37^{\circ} \mathrm{C}$ followed by addition of $10 \mu \mathrm{L}$ CCK-8 Reagent (Daeil Lab Service, Seoul, Korea).

\subsection{Statistical analysis}

Data are represented as mean \pm standard error of mean (SEM) unless otherwise indicated. Statistical analyses were performed with GraphPad Prism 5.0 (GraphPad Software, San Diego, CA, USA). Significance was determined using a two-tailed Student's $t$-test, and $p$ values $<0.05$ were considered statistically significant.

\section{RESULTS}

\subsection{Identification and sophoricoside content of S. japonica}

The structure of sophoricoside from S. japonica was assigned by nuclear magnetic resonance (NMR) and mass spectrometry (MS) analyses (data not shown). Structural elucidation and identification were carried out with MS using a JEOL JMS-600W mass spectrometer (Tokyo, Japan), and ${ }^{1} \mathrm{H}$ - and ${ }^{13} \mathrm{C}-\mathrm{NMR}$ spectra were created using a Bruker AVANCE 500 NMR spectrometer (Rheinstetten, Germany). Fast atom bombardment (FAB)-MS and NMR data were compared with values in the literature (Wu et al., 2012). The content of sophoricoside in seeds, leaves, stems, and roots of $S$. japonica by HPLC analysis are shown in Figure 1. Sophoricoside was present in the highest amount in seeds $(3.302 \mathrm{mg} / \mathrm{g} \mathrm{DW})$, followed by leaves $(0.048 \mathrm{mg} / \mathrm{g}$ DW). Sophoricoside was present in trace amounts in stems and roots of S. japonica(Table 1).

\subsection{Sophoricoside reduces allergic and asthmatic features in OVA-induced mice}

IL-3 and IL-5 are Th2 cytokines associated with allergic airway inflammation (Asquith et al, 2008; Dougan, Dranoff, \& Dougan, 2019) and sophoricoside inhibited their bioactivities (Yun et al., 2000), suggesting that allergic airway inflammation can be controlled by sophoricoside. To demonstrate this possibility, an allergic airway inflammation model was established in mice by sensitization and challenge with OVA (Figure 2A). The model was used to evaluate whether sophoricoside suppresses allergic and asthmatic symptoms. Nasal rubbing was considered a visible allergic symptom on day 26 before sacrificing mice. Induction of allergic asthma increased nasal rubbing compared with the normal group, and the behavior was decreased with administration of sophoricoside (Figure 2B). Histologic analyses showed increased infiltrating inflammatory cells in the airways and thickened airway walls in OVA-induced mice compared with normal mice. The administration of sophoricoside reduced this infiltration and thickening without cytotoxic effects in livers and spleens (Figure 2C). These results indicate that sophoricoside has anti-allergic and anti-asthmatic activities achieved through suppression of airway inflammation.

\subsection{Sophoricoside decreases AHR in OVA-induced mice}

AHR is a cardinal feature in the pathogenesis of bronchial asthma because allergic airway inflammation increases AHR and excessive bronchoconstriction in asthmatic patients (Busse, 2010). Therefore, we evaluated whether sophoricoside could improve shortness of breath and inappropriate airflow in the airways of allergic inflammation-induced asthmatic mice. Airway function was determined by calculating the Penh value using a whole-body plethysmograph. Upon inhalation of methacholine, the Penh value increased in a concentration-dependent manner in OVA-induced mice compared with normal mice. Conversely, the administration of sophoricoside decreased values from $7.19 \pm 0.62$ to $6.28 \pm 0.56$ and $3.93 \pm 0.40$ at 3 and 30 
$\mathrm{mg} / \mathrm{kg}$ compared with OVA-induced asthmatic mice at $80 \mathrm{mg} / \mathrm{mL}$ inhalation of methacholine (Figure 2D), indicating the anti-asthmatic effect of sophoricoside.

\subsection{Sophoricoside decreases recruitment of immune cells into the allergic airway}

Airway inflammation, a predominant features of allergic asthma, recruits immune cells such as eosinophils, macrophages, neutrophils, and lymphocytes (Saglani \& Lloyd, 2015). The number of various immune cells in BALF was counted to evaluate the anti-inflammatory effect of sophoricoside. Upon OVA-challenge, the number of total cells and immune cells, including macrophages, eosinophils, neutrophils, and lymphocytes, was dramatically increased compared with normal control mice. The number of recruited immune cells was effectively decreased by the administration of sophoricoside in OVA-challenged mice (Figure 3). The results indicate that sophoricoside decreases recruitment of immune cells into regions of allergic airway inflammation.

\subsection{Sophoricoside decreases release of pro-inflammatory cytokines}

Immune cells produce many inflammatory mediators that exacerbate allergic airway inflammation by inducing histamine release through mast cell activation (Pawankar et al., 2015; Hirose et al., 2017). Analysis of the released pro-inflammatory cytokines showed consistent results regarding recruitment of total cells and immune cells. Sophoricoside effectively decreased the amount of released pro-inflammatory cytokines, including IFN- $\gamma$, TNF- $\alpha$, IL-4, IL-5, IL-13, and IL-17, compared with the serum of OVA-challenged mice (Figure 4A). Interestingly, the inhibitory effect of sophoricoside on Th2 and Th17 cell-specific cytokines (IL-4, IL-5, IL-13, and IL-17) was stronger than that of Th1 cell-specific cytokines (IFN- $\gamma$ and TNF- $\alpha$ ). Consistently, the population of both IFN- $\gamma$ - and IL-4-positive cells decreased in both draining lymph nodes and BALF cells, and the population of IL-4-positive cells was more effectively decreased than that of IFN- $\gamma$-positive cells (Figure 4B and C). These results indicate that sophoricoside has anti-inflammatory activity and may have a therapeutic effect on Th cell subsets, in particular Th2 and Th17 cells.

\subsection{Sophoricoside decreases secretion of immunoglobulins in OVA-induced mice}

Elevated levels of immunoglobulins, specifically IgE, are key upstream players in allergic asthma. Therefore, anti-IgE therapy is a recommended therapeutic strategy for improving severe allergic asthma (Platts-Mills, 2001; Perez et al., 2017). The levels of total IgE and OVA-specific IgE, IgG1, and IgG2a in mice serum were measured. Higher levels of all immunoglobulins were observed in OVA-challenged mice than normal control mice. Conversely, the administration of sophoricoside effectively decreased levels of all immunoglobulins (Figure 5A-D). Consistent results were also observed by FACS analysis in BALF cells showing that elevated populations of IgE- and IgG1-positive cells with OVA-induction were significantly decreased by sophoricoside treatment (Figure 5E and F). These results indicate that sophoricoside can successfully reduce secretion of immunoglobulins, including IgE, and may improve the symptoms of allergic asthma.

\subsection{Sophoricoside reduces allergic response in PCA mice}

To further demonstrate the anti-allergic effect of sophoricoside by reducing IgE release and mast cell activation, acute allergic skin response was induced in a PCA mouse model by passive sensitization with intradermal injection of DNP-specific IgE into mice ears and intravenous challenge with soluble DNP-HSA antigen containing 5\% Evans blue dye (Figure 6A). Topical application of sophoricoside decreased mast cellmediated leakage of blood vessels, resulting in decreased amount of diffused dye (Figure 6B). Dye intensity was decreased by about $24.6 \pm 2.4 \%$ and $90.9 \pm 1.0 \%$ with 3 and $30 \mathrm{mg} / \mathrm{kg}$ sophoricoside treatment, respectively, compared with DNP-specific IgE-induced PCA mice (Figure 6C). The increased ear swelling also reduced from $16.5 \pm 1.9 \mu \mathrm{m}$ to $14.2 \pm 1.2$ and $9.4 \pm 0.8 \mu \mathrm{m}$ with 3 and $30 \mathrm{mg} / \mathrm{kg}$ sophoricoside, respectively, compared with DNP-specific IgE-induced PCA mice (Figure 6D). These results indicate that sophoricoside enacts an anti-allergic effect through suppression of IgE-mediated antibody-antigen reactions.

\subsection{Sophoricoside decreases release of histamine and lipid metabolites}

Mast cells are responsible for immediate allergic reactions initiated by cross-linking of allergens to highaffinity receptor-bound surface IgE. Released histamine and lipid metabolites, such as $\mathrm{PGD}_{2}$ and LTs, from 
IgE-primed mast cells induce allergic reactions and inflammation by recruiting immune cells such as neutrophils, eosinophils, basophils, and Th2 cells (Metcalfe et al., 2016; Schauberger et al., 2016). Therefore, the amounts of released histamine was measured to determine mast cell activation and degranulation in OVAinduced mice. OVA-induction leads to dramatically elevated histamine release in BALF compared with normal mice, and the amounts were markedly decreased by sophoricoside treatment (Figure 7A). Sophoricoside also decreased the amount of $\mathrm{LTC}_{4}$, an arachidonic acid metabolite associated with allergy and asthma, in BALF of OVA-induced mice (Figure 7B). The release of histamine and arachidonic acid metabolites, including $\mathrm{PGD}_{2}, \mathrm{LTB}_{4}$, and $\mathrm{LTC}_{4}$, in HMC-1 cells was further observed after activation with anti-DNP-IgE plus DNP-HSA. Consistent results were seen with OVA-induced mice, and treatment with sophoricoside effectively decreased histamine release in activated HMC-1 cells (Figure 7C). In addition, sophoricoside treatment decreased the amounts of $\mathrm{PGD}_{2}, \mathrm{LTB}_{4}$, and $\mathrm{LTC}_{4}$ compared to untreated HMC-1 cells (Figure $7 \mathrm{D}-\mathrm{F}$ ) without affecting cell viability (Figure $7 \mathrm{G}$ ). These results indicate that sophoricoside inhibits allergic reactions by preventing release of histamine and arachidonic acid metabolites via inhibition of mast cell activation.

\subsection{Sophoricoside suppresses $\mathrm{CD}^{+} \mathrm{T}$ cell differentiation}

Differentiated $\mathrm{CD}^{+} \mathrm{T}$ cell driven airway inflammatory responses also play an important role in allergenmediated allergic asthma. In particular, Th2 cells promote IgE-mediated sensitization, airway hyperreactivity, and eosinophilia by producing Th2 cell-specific cytokines (Ling \& Luster, 2016; , , \& 2017). Figure

4 shows results indicating that sophoricoside inhibited production of Th cell-specific cytokines in serum of OVA-challenged mice, suggesting that sophoricoside inhibits $\mathrm{CD} 4^{+} \mathrm{T}$ cell differentiation. The cytotoxic activity of sophoricoside was evaluated before determining the effect of sophoricoside on $\mathrm{CD} 4^{+} \mathrm{T}$ cell differentiation. Sophoricoside exhibited weak to no cytotoxic activity in cultured mouse CD4 ${ }^{+} \mathrm{T}$ cells in vitro at concentrations of up to $300 \mu \mathrm{M}$ for 24 to $72 \mathrm{~h}$ (Figure 8A). To confirm whether sophoricoside affects CD4 ${ }^{+} \mathrm{T}$ cell differentiation, qRT-PCR analysis was performed to determine expression of Th cell lineage-specific master regulators in cultured naïve $\mathrm{CD} 4^{+} \mathrm{T}$ cells under the appropriate differentiation conditions. Sophoricoside markedly suppressed mRNA levels of T-bet, GATA-3 and ROR $\gamma$ t, which are the master regulators for Th1, Th2, and Th17 cell subsets (Figure 8B). In addition, the mRNA levels of Th1 lineage-specific cytokine IFN- $\gamma$, Th2 lineage-specific cytokine IL-4, and Th17 lineage-specific cytokine IL-17A were effectively decreased by sophoricoside treatment (Figure 8C). Consistent with the mRNA levels of master regulators and cytokines, sophoricoside significantly decreased the amounts of released IFN- $\gamma$, TNF- $\alpha$, and IL-17A (Figure 8D). These results indicate that sophoricoside might improve Th cell-mediated airway inflammatory responses, including allergic asthma, by inhibiting CD4 ${ }^{+} \mathrm{T}$ cell differentiation.

\section{DISCUSSION}

Asthma causes serious health and socioeconomic problems worldwide because it affects a large portion of the population, from children to adults. The prevalence, hospitalization rate and mortality of asthma have markedly increased over the past half century (Dharmage, Perret, \& Custovic, 2019). Over the past few decades, asthma treatment has focused on relieving inflammation and bronchoconstriction in the respiratory system with corticosteroids, leukotriene modifiers, and anti-cholinergic agents. Recently, novel targeted therapies have been developed based on improved pathophysiologic and biologic understanding. The targets mainly include IgE, Th2 cell subset-derived cytokines, C-X-C motif chemokine receptor 2 (CXCR2), C-C motif chemokine receptor 3 (CCR3), and tyrosine kinases (Wechsler, 2018). In this study, we demonstrated the anti-allergic and anti-asthmatic effects of sophoricoside that suppress mast cell activation and $\mathrm{CD} 4^{+} \mathrm{T}$ cell differentiation.

Sophoricoside is an isoflavone substance containing genistein glycoside with a glucose moiety at position 4 . Isoflavones are widely contained in soybeans and soy protein products. They play an important role in maintaining human health via prevention of various chronic human diseases, including inflammatory disorders. Sophoricoside has been isolated from $S$. japonica, a plant of the Leguminosae family. Sophoricoside was present in the highest amount in seeds $(3.302 \mathrm{mg} / \mathrm{g} \mathrm{DW})$, followed by leaves $(0.048 \mathrm{mg} / \mathrm{g} \mathrm{DW})$, and it was 
present in trace amounts in the stems and roots of S. japonica. Since initial isolation and hormonal effects of sophoricoside were reported (Gabor, 1961), there have been few reports of its biological activities. We were interested in the inhibitory effects of sophoricoside on IL-3, IL-5, and IL-6 bioactivities (Min et al., 1999; Yun et al., 2000) and its anti-inflammatory and immunosuppressive effects (Kim et al., 2003; Kim et al., 2013; Lee et al., 2013). Specifically, IL-3, IL-5, and IL-6 are important pro-inflammatory cytokines produced from Th2 cells that play an essential role in the pathogenesis of inflammatory, allergic, and asthmatic diseases (Zhu, Yamane, \& Paul, 2010). Allergen-induced allergic and asthmatic diseases are mainly associated with type 2 inflammation, which is induced by pro-inflammatory cytokines produced from Th2 cells (Gauvreau, El-Gammal, \& O'Byrne, 2015). Results suggested that sophoricoside may affect Th2 cell-mediated allergic and asthmatic diseases.

Allergic and asthmatic diseases are commonly induced by the sensitization to and challenge with foreign antigens. The patterns of airway inflammation are caused by immune cells such as eosinophils, neutrophils, basophils, and lymphocytes controlled by $\mathrm{CD} 4^{+} \mathrm{T}$ cell-subsets, including Th1, Th2, and Th17 (Lambrecht \& Hammad, 2105). Since asthmatic inflammation is considered a hallmark of Th2 cells, we generated acute allergic and asthmatic airway inflammation by intraperitoneal sensitization and intratracheal challenge with OVA in BALB/c mice. OVA is widely used to induce experimental asthma in animals. OVA induces asthmatic airway inflammation features through $\mathrm{T}$ cell antigen receptor (TCR)-mediated Th2 type immune responses (Durrant \& Metzger, 2010). The main symptoms are characterized by influx of immune cells into the lungs, excessive production of mucus and AHR in the airway, and elevated levels of serum IgE (Holgate et al., 2015; Casaro et al., 2019). The mouse model generated by OVA showed elevated allergic and asthmatic symptoms, indicating well-induced airway inflammation in the mice. Sophoricoside treatment exhibited effective anti-allergic and anti-asthmatic activities through reduced nasal rubbing, recruitment of inflammatory cells into the lungs, AHR, and incresed levels of IgE, pro-inflammatory cytokines, histamine, and arachidonic acid metabolites. Sophoricoside also effectively decreased allergic responses such as ear swelling and mast cell-mediated leakage of blood vessels in IgE-antigen reaction-induced PCA mice. The results obtained from in vivo models indicate that sophoricoside is a promising reagent for preventing allergy and asthma.

Mast cells and eosinophils are the main effector cells of allergic inflammation. Activated mast cells contribute to secretion of histamine and newly-synthesized lipid metabolites that are responsible for production of various cytokines, chemokines, and growth factors, including IL-1, IL-3, IL-4, IL-5, IL-6, IL-8, IL-10, IL-11, IL-13, GM-CSF, TNF- $\alpha$, transforming growth factor-beta (TGF- $\beta$ ), basic fibroblast growth factor (b-FGF), nerve growth factor (NGF), and vascular endothelial growth factor (VEGF) (Minai-Fleminger \& Levi-Schaffer, 2009; Stone, Prussin, \& Metcalfe, 2010). IgE and Th2 subset signature cytokines IL-4 and IL-13 play an important role in maintaining mast cell homeostasis, and released mast cell metabolites recruit Th2 cells and eosinophils to inflammatory sites to promote allergic process (Leung, Beukema, \& Shen, 2015; McLeod, Baker, \& Ryan, 2015; Simon et al., 2020). To evaluate whether sophoricoside influences mast cell activation, we determined the amounts of released histamine and arachidonic acid metabolites in OVA-induced mice and human mast cells stimulated with IgE plus antigen. Sophoricoside decreased the levels of released histamine and arachidonic acid metabolites in OVA-induced mice and mast cells, indicating an inhibitory effect of sophoricoside on mast cell activation and reduced allergic responses. Histamine and arachidonic acid metabolites released from IgE-primed mast cells recruit immune cells, such as eosinophils, neutrophils, basophils, and Th2 cells (Metcalfe et al., 2016; Schauberger et al., 2016). Based on these signaling networks, the correlation between mast cell activation, immune cell recruitment, and CD4 ${ }^{+} \mathrm{T}$ cell differentiation is tightly correlated with exacerbation of allergic inflammation. In this pathogenic circumstance, sophoricoside reduced allergic airway inflammation by inhibition of signaling loops.

Naïve CD4 ${ }^{+}$T cell-derived effector subsets mainly include Th1, Th2, Th17, and Treg, which play important roles in direct immune responses. These cells aggravate allergic airway inflammation by producing lineagespecific signature cytokines and recruiting immune cells to inflammatory sites (Zhu, Yamane, \& Paul, 2010; Akdis \& Akdis, 2014; Lambrecht \& Hammad, 2015). Interestingly, sophoricoside more strongly inhibited the release of Th2 and Th17 specific cytokines, such as IL-4, IL-5, IL-13, and IL-17, compared to Th1 specific 
cytokines, such as IFN- $\gamma$ and TNF- $\alpha$. Consistent results were also observed within vitro differentiation experiments of naïve CD4 ${ }^{+} \mathrm{T}$ cells into Th1, Th2, and Th17 cell subsets. Sophoricoside also inhibited the mRNA levels of master regulators, T-bet, GATA-3, and ROR $\gamma t$, for Th1, Th2, and Th17 cell subsets, respectively, as well as the production and mRNA levels of Th cell-specific cytokines. The inhibitory effects of sophoricoside on Th2 and Th17 cell subsets were slightly stronger than those on the Th1 cell subset.

In summary, we isolated sophoricoside from mature seeds of $S$. japonica to investigate its anti-allergic and anti-asthmatic activities in animal models. There was higher sophoricoside content in mature seeds than in leaves, stems, and roots. Sophoricoside reduced allergic and asthmatic responses in OVA-induced allergic airway inflammation and PCA models by inhibiting mast cell activation and CD4 ${ }^{+} \mathrm{T}$ cell differentiation. Our results imply that sophoricoside may be a useful therapeutic candidate for treating allergic diseases, including allergic asthma.

\section{ACKNOWLEDGEMENTS}

This work was supported by grants from Natural Product Institute of Science and Technology (www.nist.re.kr), Anseong 17546, Republic of Korea.

\section{CONFLICT OF INTEREST}

The authors declare no conflicts of interest.

\section{AUTHOR CONTRIBUTIONS}

B.H.K. and D.G.L. designed and performed experiments and analyzed data. D.G.L. and S.L. isolated and purified sophoricoside. B.H.K., D.G.L., and S.L. wrote the manuscript. All authors discussed the results, commented and approved the final manuscript.

\section{REFERENCES}

Abdallah, H. M., Al-Abd, A. M., Asaad, G. F., Abdel-Naim, A. B., \& El-halawany, A. M. (2014). Isolation of antiosteoporotic compounds from seeds of Sophora japonica.PLoS One , 9 (6), e98559. doi: 10.1371/journal.pone.0098559

Akdis, M., \& Akdis, C. A. (2014). Mechanisms of allergen-specific immunotherapy: multiple suppressor factors at work in immune tolerance to allergens. Journal of Allergy and Clinical Immunology, 133 (3), 621631. doi: $10.1016 /$ j.jaci.2013.12.1088

Asquith, K. L., Ramshaw, H. S., Hansbro, P. M., Beagley, K. W., Lopez, A. F., \& Foster, P. S. (2008). The IL-3/IL-5/GM-CSF common receptor plays a pivotal role in the regulation of Th2 immunity and allergic airway inflammation. Journal of Immunology, 180 (2), 1199-1206.

Busse, W. W. (2010). The relationship of airway hyperresponsiveness and airway inflammation: Airway hyperresponsiveness in asthma: its measurement and clinical significance. Chest, 138 (2 Suppl), 4S-10S. doi: $10.1378 /$ chest.10-0100

Casaro, M., Souza, V. R., Oliveira, F. A., \& Ferreira, C. M. (2019). OVA-induced allergic airway inflammation mouse model.Methods in Molecular Biology, 1916 , 297-301. doi: 10.1007/978-1-4939-8994-2_28

Dharmage, S. C., Perret, J. L., \& Custovic, A. (2019). Epidemiology of asthma in children and adults. Frontiers in Pediatrics , 7 , 246. doi: 10.3389/fped.2019.00246

Dougan, M., Dranoff, G., \& Dougan, S. K. (2019). GM-CSF, IL-3, and IL-5 family of cytokines: Regulators of inflammation. Immunity,50 (4), 796-811. doi: 10.1016/j.immuni.2019.03.022

Durrant, D. M. \& Metzger, D. W. (2010). Emerging roles of T helper subsets in the pathogenesis of asthma.Immunological Investigation, 39 (4-5), 526-549. doi: 10.3109/08820131003615498 
El-Halawany, A. M., Chung, M. H., Abdallah, H. M., Nishihara, T., \& Hattori, M. (2010). Estrogenic activity of a naringinase-treated extract of Sophora japonica cultivated in Egypt. Pharmaceutical Biology , 48 (2), 177-181. doi: 10.3109/13880200903062663

Fahy, J. V. (2015). Type 2 inflammation in asthma-present in most, absent in many. Nature Reviews Immunology , 15 (1), 57-65. doi: 10.1038/nri3786

Gabor, M. (1961). The hormonal effect of an isoflavone derivative (sophoricoside).Kiserl Orvostud, 13 , 133-134.

Gauvreau, G. M., El-Gammal, A. I., \& O'Byrne, P. M. (2015). Allergen-induced airway responses.European Respiratory Journal , 46 (3), 819-831. doi: 10.1183/13993003.00536-2015

Hirose, K., Iwata, A., Tamachi, T., \& Nakajima, H. (2017). Allergic airway inflammation: Key players beyond the Th2 cell pathway.Immunological Reviews, 278 (1), 145-161. doi: 10.1111/imr.12540

Holgate, S. T., Wenzel, S., Postma, D. S., Weiss, S. T., Renz, H., \& Sly, P. D. (2015). Asthma.Nature Reviews Disease Primers, 1 , 15025. doi: 10.1038/nrdp.2015.25

Jung, S. H., Cho, S. H., Dang, T. H., Lee, J. H., Ju, J. H., ... Kim, Y. (2003). Structural requirement of isoflavonones for the inhibitory activity of interleukin-5. European Journal of Medicinal Chemistry, 38 (5), 537-545.

Kim, B. H., Chung, E. Y., Min, B. K., Lee, S. H., Kim, M. K., .. Kim, Y. (2003). Anti-inflammatory action of legume isoflavonoid sophoricoside through inhibition on cyclooxygenase-2 activity. Planta Medica , 69 (5), 474-476.

Kim, B. H., Chung, E. Y., Ryu, J. C., Jung, S. H., Min, K. R., \& Kim, Y. (2003). Anti-inflammatory mode of isoflavone glycoside sophoricoside by inhibition of interleukin- 6 and cyclooxygenase- 2 in inflammatory response.Archives of Pharmacal Research , 26 (4), 306-311.

Kim, B. H., Oh, I., Kim, J. H., Jeon, J. E., Jeon, B., .. Kim, T. Y. (2014). Anti-inflammatory activity of compounds isolated from Astragalus sinicus L. in cytokine-induced keratinocytes and skin. Experimental $\mathscr{E}$ Molecular Medicine , 46 , e87. doi: 10.1038/emm.2013.157

Kim, S. J., Lee, G. Y., Jung, J. W., Oh, S. R., Ahn, E. M., ... Um, J. Y. (2013). The ameliorative effect of sophoricoside on mast cell-mediated allergic inflammation in vivo and in vitro. Molecules ,18 (5), 6113-6127. doi: 10.3390/molecules18056113

Lambrecht, B. N. \& Hammad, H. (2015). The immunology of asthma.Nature Immunology , 16 (1), 45-56. doi: $10.1038 /$ ni.3049

Lambrecht, B. N., Hammad, H., \& Fahy, J. V. (2019). The cytokines of asthma.Immunity, 50 (4), 975-991. doi: $10.1016 /$ j.immuni.2019.03.018

Lee, H. G., Cho, N. C., Jeong, A. J., Li, Y. C., Rhie, S. J., .. Kim, B. H. (2016). Immunomodulatory activities of the benzoxathiole derivative BOT-4-one ameliorate pathogenic skin inflammation in mice. Journal of Investigative Dermatology , 136 (1), 107-116. doi: 10.1038/JID.2015.384

Lee, H. K., Kim, H. S., Kim, Y. J., Kim, J. S., Park, Y. S., .. Han, S. B. (2013). Sophoricoside isolated from Sophora japonica ameliorates contact dermatitis by inhibiting NF- $x \mathrm{~B}$ signaling in B cells. International Immunopharmacology , 15 (3), 467-473. doi: 10.1016/j.intimp.2013.01.025

Leung, J., Beukema, K. R., \& Shen, A. H. (2015). Allergic mechanisms of Eosinophilicoesophagitis. Best Practice 83 Research Clinical Gastroenterology , 29 (5), 709-720. doi: 10.1016/j.bpg.2015.09.012

Li, W., \& Lu, Y. (2018). Hepatoprotective effects of sophoricoside against fructose-induced liver injury via regulating lipid metabolism, oxidation, and inflammation in mice.Journal of Food Science , 83 (2), 552-558. doi: $10.1111 / 1750-3841.14047$ 
Ling, M. F., \& Luster, A. D. (2016). Allergen-specific CD4(+) T cells in human asthma.Annals of the American Thoracic Society, 13 (Suppl 1), S25-S30. doi: 10.1513/AnnalsATS.201507-431MG

Marshall, J. S., Warrington, R., Watson, W., \& Kim, H. L. (2018). An introduction to immunology and immunopathology.Allergy Asthma and Clinical Immunology , 14 (Supple 2), 49. doi: 10.1186/s13223-0180278-1

McLeod, J. J., Baker, B., \& Ryan, J. J. (2015). Mast cell production and response to IL-4 and IL13.Cytokine, 75 (1), 57-61. doi: 10.1016/j.cyto.2015.05.019

Metcalfe, D. D., Pawankar, R., Ackerman, S. J., Akin, C., Clayton, F., .. Triggiani, M. (2016). Biomarkers of the involvement of mast cells, basophils and eosinophils in asthma and allergic disease. World Allergy Organization Journal, 9 , 7. doi: 10.1186/s40413-016-0094-3

Min, B., Oh, S. R., Lee, H. K., Takatsu, K., Chang, I. M., .. Kim, Y. (1999). Sophoricoside analogs as the IL-5 inhibitors from Sophora japonica. Planta Medica, 65 (5), 408-412.

Minai-Fleminger, Y., \& Levi-Schaffer, F. (2009). Mast cells and eosinophils: The two key effector cells in allergic inflammation. Inflammation Research , 58 (10), 631-628. doi: 10.1007/s00011-009-0042-6

Muehling, L. M., Lawrence, M. G., \& Woodfolk, J. A. (2017). Pathogenic CD4 ${ }^{+}$T cells in patients with asthma. Journal of Allergy and Clinical Immunology, 140 (6), 1523-1540. doi: 10.1016/j.jaci.2017.02.025.

Papi, A., Brightling, C., Pedersen, S. E., \& Reddel, H. K. (2018). Asthma.Lancet,391 (10122), 783-800. doi: 10.1016/S0140-6736(17)33311-1

Pawankar, R., Hayashi, M., Yamanishi, S., \& Igarashi, T. (2015). The paradigm of cytokine networks in allergic airway inflammation. Current Opinion in Allergy and Clinical Immunology, 15 (1), 41-48. doi: 10.1097/ACI.0000000000000129

Perez, E. E., Orange, J. S., Bonilla, F., Chinen, J., Chinn, I. K., .. Ballow, M. (2017). Update on the use of immunoglobulin in human disease: A review of evidence. Journal of Allergy and Clinical Immunology, 139 (3S), S1-S46. doi: 10.1016/j.jaci.2016.09.023

Platts-Mills, T. A. (2001). The role of immunoglobulin E in allergy and asthma.American Journal of Respiratory and Critical Care Medicine, 164 (8 Pt 2), S1-S5.

Quirt, J., Hildebrand, K. J., Mazza, J., Noya, F., \& Kim, H. (2018). Asthma.Allergy Asthma and Clinical Immunology , 14 (Supple 2), 50. doi: 10.1186/s13223-018-0279-0

Ray, A., Raundhal, M., Oriss, T. B., Ray, P., \& Wenzel, S. E. (2016). Current concepts of severe asthma.Journal of Clinical Investigation, 126 (7), 2394-2403. doi: 10.1172/JCI84144

Saglani, S., \& Lloyd, C. M. (2015). Novel concepts in airway inflammation and remodeling in asthma.European Respiratory Journal, 46 (6), 1796-1804. doi: 10.1183/13993003.01196-2014

Saltini, C., Hance, A. J., Ferrans, V. J., Basset, F., Bitterman, P. B., \& Crystal, R. G. (1984). Accurate quantification of cells recovered by bronchoalveolar lavage. American Review of Respiratory Disease , 130 (4), 650-658.

Schauberger, E., Peinhaupt, M., Cazares, T., \& Lindsley, A.W. (2016). Lipid mediators of allergic disease: Pathways, treatments, and emerging therapeutic targets.Current Allergy and Asthma Reports, 16 (7), 48. doi: $10.1007 / \mathrm{s} 11882-016-0628-3$

Simon, H. U., Yousefi, S., Germic, N., Arnold, I. C., Haczku, A., .. Rosenberg, H. F. (2020). The cellular functions of eosinophils: Collegium internationale allergologicum (CIA) update 2020.International Archives of Allergy and Immunology, 181 (1), 11-23. doi: 10.1159/000504847

Stone, K. D., Prussin, C., \& Metcalfe, D. D. (2010). IgE, mast cells, basophils, and eosinophils. Journal of Allergy and Clinical Immunology,125 (Suppl 2), S73-S80. doi: 10.1016/j.jaci.2009.11.017. 
Wechsler, M. E. (2018). Current and emerging biologic therapies for asthma and COPD.Respiratory Care, 63 (6), 699-707. doi: 10.4187/respcare.06322

Wu, C., Luan, H., Wang, S., Zhang, X., Wang, R., .. Chen, X. (2013). Modulation of lipogenesis and glucose consumption in HepG2 cells and C2C12 myotubes by sophoricoside. Molecules , 18 (12), 1562415635. doi: 10.3390/molecules 181215624

Wu, J., Yang, X., Ge, J., Zhang, Y., Wu, L., ... Zhang, X. (2012). Biotransformation of sophoricoside in Fructus sophorae by the fungusSchizophyllum commune. Bioresource Technology, 111 , 496-499. doi: 10.1016/j.biortech.2012.02.038

Yun, J., Lee, C. K., Chang, I. M., Takatsu, K., Hirano, T., .. Kim, Y. (2000). Differential inhibitory effects of sophoricoside analogs on bioactivity of several cytokines. Life Sciences , 67 (23), 2855-2863.

Zhu, J., Yamane, H., \& Paul, W. E. (2010). Differentiation of effector CD4 T cell populations (*).Annual Review of Immunology, 28 , 445-489. doi: 10.1146/annurev-immunol-030409-101212

Zinellu, E., Piras, B., Ruzittu, G. G. M., Fois, S. S., Fois, A. G., \& Pirina, P. (2019). Recent advances in inflammation and treatment of small airways in asthma.International Journal of Molecular Sciences , 20 (11), E2617. doi: 10.3390/ijms20112617

\section{FIGURE LEGENDS}

Figure 1. Chemical structure of sophoricoside and HPLC chromatograms. Chemical structure of sophoricoside and its HPLC chromatogram (A). HPLC chromatograms of EtOH extracts of seeds (B), leaves (C), stems (D), and roots (E) of S. japonica . * indicates sophoricoside.

Figure 2. Sophoricoside reduces allergic airway inflammation in OVA-induced mice. Experimental scheme for OVA-induced allergic airway inflammation in BALB/c mice (A). Number of nasal rubbing events was determined for 10 min on the day 26 (B). Histology of lung (upper), liver (middle), and spleen (bottom) was assessed with H\&E staining. Scale bars: 100 or $200 \mu \mathrm{m}$ or $1.0 \mathrm{~mm}$ (C). AHR was measured by Penh value under inhalation with indicated concentrations of methacholine (D). \#p<0.005 versus vehicle-treated group; ${ }^{* *} p<0.005$ versus OVA-induced group $(n=6)$. SOP, sophoricoside.

Figure 3. Sophoricoside inhibits recruitment of immune cells into allergic airway in OVAinduced mice. Cells prepared from BALF were applied to a slide by cytospinning and stained with Diff-Quick or Wright-Giemsa solution. The number of total cells, macrophages, eosinophils, neutrophils, and lymphocytes was determined by counting within a $1 \mathrm{~mm}^{2}$ area (A). $\# p<0.005$ versus vehicle-treated group; ${ }^{* *} p<0.005$ versus OVA-induced group $(n=6)$. (B and C) Cells were stained with either Diff-Quick or Wright-Giemsa solution, and total cells (B) and eosinophils (C) were observed under light microscope. Scale bars: 50 or $20 \mu \mathrm{m}$. SOP, sophoricoside.

Figure 4. Sophoricoside inhibits release of pro-inflammatory cytokines in OVA-induced mice. Amounts of pro-inflammatory cytokines were measured by ELISA from serum of OVA-induced mice (A). $\# p<0.005$ versus vehicle-treated group; ${ }^{*} p<0.05$ and ${ }^{* *} p<0.005$ versus OVA-induced group $(n=6)$. (B and C) Draining lymph node cells (B) and BALF cells (C) were stained with PE-conjugated anti-CD4 antibody, APC-conjugated anti-IL-4 antibody, or FITC-conjugated anti-IFN- $\gamma$ antibody, and populations of CD4-, IL-4-, and IFN- $\gamma$-positive cells were counted by FACS analysis. SOP, sophoricoside.

Figure 5. Sophoricoside inhibits secretion of immunoglobulins in OVA-induced mice. (A-D) Amounts of total IgE (A), OVA-specific IgE (B), OVA-specific IgG1 (C), and OVA-specific IgG2a (D) were measured by ELISA from serum of OVA-induced mice. $\# p<0.005$ versus vehicle-treated group; ${ }^{* *} p<$ 0.005 versus OVA-induced group $(n=6)$. ( $\mathrm{E}$ and $\mathrm{F}$ ) Total cells prepared from BALF were stained with FITC-conjugated anti-IgE or PE-conjugated anti-IgG1 antibody, and populations of IgE- and IgG1-positive cells were counted by FACS analysis. SOP, sophoricoside.

Figure 6. Sophoricoside inhibits allergic response in PCA mice.Experimental scheme for PCA 
experiment in C57BL/6 mice (A). (B-D) Mice were passively sensitized with 100 ng anti-DNP IgE. Twentyfour hours after intradermal injection of anti-DNP IgE, sophoricoside was topically applied to mouse ears $1 \mathrm{~h}$ before intravenous injection of $100 \mu \mathrm{g}$ DNP-HSA containing 5\% Evans' blue dye. One hour after DNP-HSA injection, images of the ears (B), amounts of Evans blue dye (C), and ear swelling (D) were measured. \#p $<0.005$ versus vehicle-treated group; ${ }^{*} p<0.05$ and ${ }^{* *} p<0.005$ versus $\operatorname{IgE} /$ antigen-induced group $(n=5)$. SOP, sophoricoside.

Figure 7. Sophoricoside inhibits release of histamine and arachidonic acid metabolites. (A and B) Amounts of histamine (A) and $\mathrm{LTC}_{4}$ (B) were measured by ELISA from BALF. \# $p<0.005$ versus vehicle-treated group; ${ }^{* *} p<0.005$ versus OVA-induced group $(n=6)$. (C-F) HMC-1 cells were sensitized with $1 \mu \mathrm{g} / \mathrm{mL}$ anti-DNP-IgE overnight. Cells were washed twice and treated with sophoricoside for $30 \mathrm{~min}$, followed by activation with DNP-HAS $(10 \mu \mathrm{g} / \mathrm{mL})$. Amounts of histamine (C), $\mathrm{PGD}_{2}(\mathrm{D}), \mathrm{LTB}_{4}(\mathrm{E})$, and $\mathrm{LTC}_{4}(\mathrm{~F})$ were measured by ELISA. $\# p<0.005$ versus vehicle-treated group; ${ }^{*} p<0.05$ and ${ }^{* *} p<0.005$ versus IgE/antigen-induced group $(n=3)$. (G) HMC-1 cells were incubated with various concentrations of sophoricoside for $24 \mathrm{~h}$, and their viability was measured. SOP, sophoricoside.

Figure 8. Sophoricoside inhibits $\mathbf{C D} 4^{+} \mathbf{T}$ cell differentiation. $\mathrm{CD} 4^{+} \mathrm{T}$ cells were incubated with various concentrations of sophoricoside for $24-72 \mathrm{~h}$, and their viability was measured (A). (B-D) Naïve CD4 ${ }^{+}$ $\mathrm{T}$ cells were differentiated under appropriate differentiation conditions for 5 days in the presence or absence of sophoricoside $(30 \mu \mathrm{M})$, as described in METHODS. Total RNA was isolated, and qRT-PCR was performed to analyze levels of $\mathrm{CD}^{+}{ }^{+} \mathrm{T}$ cell lineage-specific master regulators (B) and cytokines $(\mathrm{C})$. Cytokine amounts were measured by ELISA using cultured supernatants (D). ${ }^{* *} p<0.005$ versus differentiated group $(n=3)$. SOP, sophoricoside.

\section{BULLET POINT SUMMARY}

\section{What is already known}

- Sophoricoside inhibits IL-3, IL-5, and IL-6 bioactivity and has anti-inflammatory activity.

- IL-3 and IL-5 are Th2 cell-specific signature cytokines associated with allergic inflammation.

\section{What this study adds}

- Sophoricoside improved allergic asthma and allergic responses in OVA-induced and PCA models.

- Sophoricoside inhibited mast cell activation and CD4 ${ }^{+} \mathrm{T}$ cell differentiation.

\section{What is the clinical significance}

Sophoricoside has therapeutic potential in allergic diseases, including allergic asthma.

\section{Hosted file}

SOP_BJP_table.docx available at https://authorea.com/users/313085/articles/443705-sophoricosidefrom-sophora-japonica-ameliorates-allergic-asthma-by-preventing-mast-cell-activation-andcd4-t-cell-differentiation-in-ovalbumin-induced-mice

\section{Hosted file}

SOP_BJP_figure.pptx available at https://authorea.com/users/313085/articles/443705-sophoricosidefrom-sophora-japonica-ameliorates-allergic-asthma-by-preventing-mast-cell-activation-andcd4-t-cell-differentiation-in-ovalbumin-induced-mice 